\title{
Circulation in High Reynolds Number Isotropic Turbulence is a Bifractal
}

\author{
Kartik P. Iyer, ${ }^{1}$ Katepalli R. Sreenivasan, ${ }^{1,2, *}$ and P. K. Yeung ${ }^{3}$ \\ ${ }^{1}$ Department of Mechanical and Aerospace Engineering, New York University, \\ New York, New York 11201, USA \\ ${ }^{2}$ Department of Physics and the Courant Institute of Mathematical Sciences, \\ New York University, New York, New York 11201, USA \\ ${ }^{3}$ Schools of Aerospace Engineering and Mechanical Engineering, \\ Georgia Institute of Technology, Atlanta, Georgia 30332, USA
}

\begin{abstract}
(Received 15 February 2019; revised manuscript received 14 August 2019; published 4 October 2019)
The turbulence problem at the level of scaling exponents is hard in part because of the multifractal scaling of small scales, which demands that each moment order be treated and understood independently. This conclusion derives from studies of velocity structure functions, energy dissipation, enstrophy density (that is, square of vorticity), etc. However, it is likely that there exist other physically pertinent quantities with less complex statistical structure in the inertial range, potentially resulting in huge simplifications in the turbulence theory. We show that velocity circulation around closed loops is such a quantity. By using a large database of isotropic turbulence, generated from numerical simulations of the Navier-Stokes equations over a wide range of Reynolds numbers, we show that circulation exhibits, to excellent accuracy, a bifractal behavior at the highest Reynolds number considered: space filling for low-order moments, close but not identical to the 1941 paradigm of Kolmogorov, and a monofractal with a dimension of about 2.2 for higher orders. This change in character, occurring around the third moment for the highest Reynolds number considered here, is reminiscent of a "phase transition." We explore the possibility that the transition point moves to higher-order moments as the Reynolds numbers increases-even though one may continue to regard the structure as bifractal for moments of sufficiently high order. We confirm that the circulation properties depend essentially on the area of the loop, not its shape, and that the relevant contour area in figure-eight loops is the scalar area and not the vector area. These results demonstrate an intrinsic simplicity in the statistical structure of turbulence when considering circulation around closed loops, thus motivating a paradigm shift in turbulence research.
\end{abstract}

DOI: 10.1103/PhysRevX.9.041006

\section{INTRODUCTION}

From Leonardo da Vinci's half-a-millennium-old drawings of turbulent motions in the river Arno [1] to their visualizations on modern-day computers [2], evidence abounds that turbulent motion comprises organized structures, often evocatively described as "eddies" and "vortices." On the other hand, both phenomenological and analytical theories of turbulence $[3,4]$ have largely focused on multipoint correlators of velocity, whose connection to the physical structures is not always clear. Furthermore, the most obvious multipoint correlators are best described as multifractals with an infinity of independent exponents

*krs3@nyu.edu

Published by the American Physical Society under the terms of the Creative Commons Attribution 4.0 International license. Further distribution of this work must maintain attribution to the author(s) and the published article's title, journal citation, and DOI.
Subject Areas: Fluid Dynamics, Nonlinear Dynamics, Statistical Physics

[5-8], which makes the problem very difficult to explore analytically. In this paper, we show that the velocity circulation around closed loops, besides providing a plausible link between vortical structures and statistical objects, has a simple bifractal structure in the inertial range (IR) - this being the range of scales that is simultaneously far from the forcing and dissipation scales. We find that the circulation moments up to about order 3 are close to Kolmogorov's 1941 paradigm [3], henceforth denoted by $\mathrm{K} 41$, arising from a space-filling set of dimension three. Even though the departures from K41 are small, they seem to be real. For higher orders, the moments live on a fractal set with a dimension of about 2.2. We comment on possible Reynolds number effects and the implications for the intermittency of vorticity and velocity increments.

For reference, the circulation around a loop of linear dimension $r$ is defined as

$$
\Gamma_{r} \equiv \oint_{C} \mathbf{u}(\mathbf{l}) \cdot d \mathbf{l}=\oiint_{A} \omega \cdot \hat{\mathbf{n}} d A
$$


where $C$ is the boundary of a loop of area $A\left(\sim r^{2}\right)$, $\mathbf{u}$ is the velocity, $d \mathbf{l}$ is an elemental length along $C, \omega \equiv \nabla \times \mathbf{u}$ is the vorticity, and $\hat{\mathbf{n}} d A$ is an elemental area of $A$ in the direction of the unit normal $\hat{\mathbf{n}}$. Migdal [9] initiated the statistical theory of velocity circulation around closed Eulerian loops by recasting the incompressible NavierStokes equations via a reduced Hopf generating functional [10]. He argued, on the basis of WKB asymptotics, that the tails of the probability density function (PDF) depend only on the minimal area circumscribed by a simply connected loop, and not on its actual shape, as long as it is entirely contained in IR. This is the area rule. Another question posed by Migdal was whether, for a figure-eight loop, the circulation statistics depend on the scalar sum of the two subareas enclosed by the loop, or on their vectorial sum as would be the case if one used the standard sign convention for loop area. Within IR, he also reasoned that the PDF of $\Gamma_{r}$ might be consistent with K41. Since $\Gamma_{r}$ has the dimension of a characteristic velocity times the length scale $r$, and the velocity increment in K41 scales as $r^{1 / 3}$, he expected the PDF of $\Gamma_{r}^{3} / r^{4}$ to possess a universal shape in IR. A more general scaling would be $\Gamma_{r}^{2 k} / r^{4 k-2}$, where $k$ is arbitrary [10]. Migdal did not have conclusive thoughts on the scaling of high-order circulation moments.

This theoretical framework was soon explored by a small number of experimental and numerical papers [11-15]. Because they were all limited to low Reynolds numbers, the verification of the area rule was stymied by the modest extent of IR, and, because each of them considered only one Reynolds number, none of them could extrapolate their results to the infinite-Reynolds-number limit. Further, the inferences drawn from them were ambiguous because the experimental flows were not all homogeneous. It is thus not a great surprise that these earlier studies did not agree quantitatively among themselves. One inference drawn by all these early studies was that circulation is also highly intermittent, just as the velocity increments are, and display multifractal scaling with no unique scaling for all moments, making no new insights possible. We assess these properties persuasively here by taking recourse to direct numerical simulation (DNS) data of statistically stationary, homogeneous, and isotropic turbulence in a periodic box over a wide range of Reynolds numbers [16,17].

We first show that the area rule applies to a good approximation to the entire PDF of circulation around planar loops, not just its tails, and that it depends on the scalar sum of the two subareas for figure-eight loops. We also show that circulation at the level of low-order moments is space filling and scales close to $\mathrm{K} 41$, but that it resides on a fractal set of dimension 2.2 for moments of higher orders than about 3 . This simple behavior contrasts a highly intermittent, multifractal structure of velocity increments, dissipation, and vorticity at all orders. Thus, the result goes a considerable distance in addressing the question: Among the many statistical variables that can be used to describe inertial range intermittency, is there one that does not demand an infinity of independent exponents?

\section{DATA}

The DNS data used in this work have been acquired by solving the incompressible Navier-Stokes equations,

$$
\partial \mathbf{u} / \partial t+(\mathbf{u} \cdot \nabla) \mathbf{u}=-\nabla(p / \rho)+\nu \nabla^{2} \mathbf{u}+\mathbf{f},
$$

where $\mathbf{u}$ is the solenoidal velocity field $(\nabla \cdot \mathbf{u}=0), p$ is pressure, $\rho$ is fluid density, $\nu$ is the kinematic viscosity, and $\mathbf{f}$ is the forcing term that maintains a stationary state $[18,19]$. We use Fourier pseudospectral calculations [20] on a periodic domain of size $(2 \pi)^{3}$ with an explicit secondorder Runge-Kutta integration in time. A combination of phase shifting and truncation is used to reduce aliasing errors, where the highest resolved wave number $k_{\max }=$ $\sqrt{2} N / 3$ and $N$ is the number of grid points in one direction. As in earlier simulations $[16,19,21]$, the resolution was held around $k_{\max } \eta=1$, where $\eta$ is nominally the smallest scale of motion (defined more precisely later). Recently in Ref. [17], it has been pointed out that the spatial and temporal resolution are more stringent at higher Reynolds numbers, and so improved resolution was sought in some instances. See Table I, which lists some flow parameters of general interest.

One other comment is useful. Circulation $\Gamma_{r}$ around a loop of side $r$ is calculated using the second equality in Eq. (1), which follows from the Stokes theorem, as the two-dimensional local average of vorticity using the algorithm given in Ref. [22]. Averages were performed over the whole $N^{3}$ simulation along the three Cartesian directions. Statistics of $\Gamma_{r}$ were also obtained using the loop integration of Eq. (1), by means of cubic splines for improved accuracy, and excellent confirmation of the area

TABLE I. Isotropic DNS database. $N^{3}$ is the number of points on a $L_{0}^{3}$ grid with $L_{0}=2 \pi$ units, $R_{\lambda} \equiv u^{\prime} \lambda / \nu$ is the Taylor-scale Reynolds number where $u^{\prime}$ is the root-mean-square velocity fluctuation, $\lambda \equiv u^{\prime} / \sqrt{\left\langle(\partial u / \partial x)^{2}\right\rangle}$ is the Taylor microscale, $\nu$ is the kinematic viscosity, $L \approx L_{0} / 5$ is the integral scale, $\eta \equiv$ $\left(\nu^{3} /\langle\epsilon\rangle\right)^{1 / 4}$ is the Kolmogorov scale, $\Delta x=L_{0} / N$ is the grid spacing. Results have been averaged over a time span of at least 10 large-eddy timescales $\left(L / u^{\prime}\right)$, except for the $16384^{3}$ data for which the averaging time is much shorter.

\begin{tabular}{lrrcc}
\hline \hline$N^{3}$ & $R_{\lambda}$ & $L / \eta$ & $\Delta x / \eta$ & $\langle\epsilon\rangle L / u^{\prime 3}$ \\
\hline $256^{3}$ & 140 & 108 & 2.1 & 0.44 \\
$512^{3}$ & 240 & 226 & 2.1 & 0.42 \\
$2048^{3}$ & 400 & 446 & 1.1 & 0.41 \\
$4096^{3}$ & 650 & 898 & 1.1 & 0.39 \\
$8192^{3}$ & 1300 & 2514 & 1.5 & 0.38 \\
$16384^{3}$ & 1300 & 2522 & 0.8 & 0.39 \\
\hline \hline
\end{tabular}


integral results were obtained for $r / \eta>5$ (see the Appendix A for details).

\section{RESULTS}

\section{A. Area rules}

To be specific, we provide the following definitions. The longitudinal velocity increment is defined as $\Delta_{r} u \equiv$ $u(x+r)-u(x)$, where the velocity component $u$ and the separation distance $r$ are both taken in the same direction. For this paper, the inertial range is defined as that range where the circulation moments display power-law behavior; it is essentially the range where the normalized third-order velocity structure function $\left\langle\left(\Delta_{r} u\right)^{3}\right\rangle / r\langle\epsilon\rangle,\langle\epsilon\rangle$ being the global mean value of the energy dissipation rate, is equal [23] to within $\pm 2.5 \%$ of the exact theoretical value of $-4 / 5$ [24]. We have indeed experimented within limits the sensitivity of the results to the precise definition of IR, and found that the results presented are quite robust.

Figure 1 shows circulation traces for square loops in IR, normalized by its standard deviation, at the microscale Reynolds number $R_{\lambda}=1300$. Two different inertial separations $r$, corresponding to the lower end and the middle of the inertial range plateau in $-\left\langle\left(\Delta_{r} u\right)^{3}\right\rangle / r\langle\epsilon\rangle$, are shown. These particular signals do not show frequent excursions to very high values (unlike vorticity, for example).

Figure 2 shows the PDFs of $\Gamma_{A}$ for a number of rectangular loops of the same area but differing aspect ratios. As long as one of the sides of the loop lies outside IR, the PDFs do not collapse, but they do collapse to a very good accuracy when all sides of the rectangle lie within IR. Even though the result will have to be confirmed for loops of different shapes and for nonplanar configurations, this
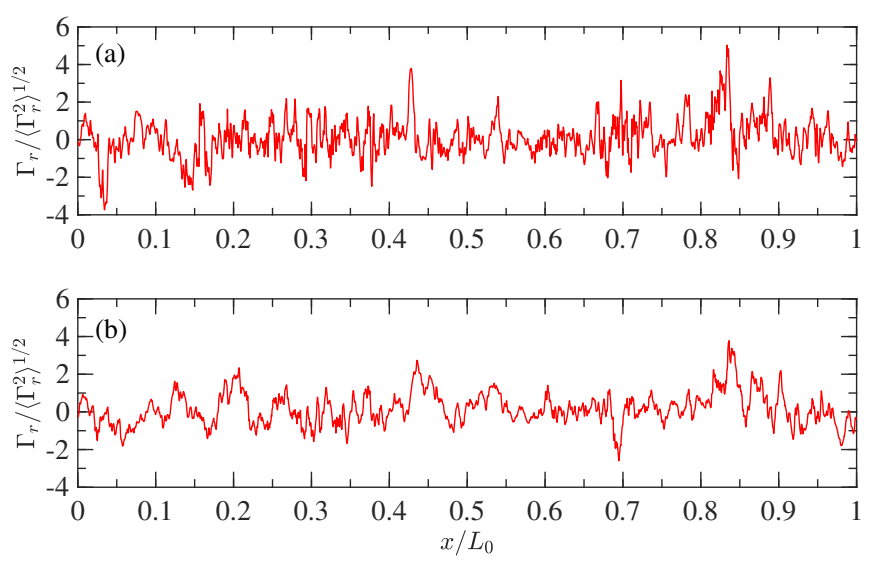

FIG. 1. Typical circulation traces in the inertial range, for $R_{\lambda}=1300$ (computational domain $8192^{3}$ ) along an arbitrary edge of the cube of length $L_{0}$. The linear sizes $r$ of the loops are (a) $r / \eta=50$ and (b) $r / \eta=150$, corresponding, respectively, to the lower end and middle of the $4 / 5$ plateau in the normalized third-order structure function [24].

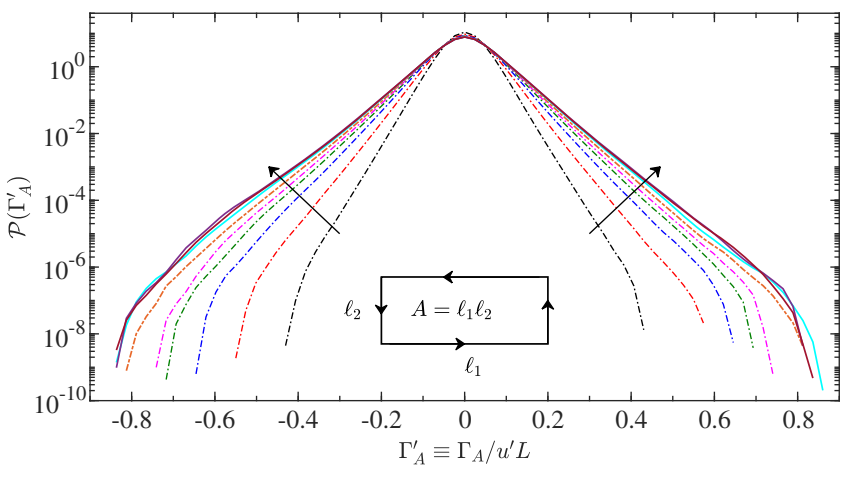

FIG. 2. Probability density function $\mathcal{P}$ of normalized circulation around loops with fixed area, $A=\ell_{1} \ell_{2}=4646 \eta^{2}$, but with different edge lengths, calculated for the $4096^{3}$ data at $R_{\lambda}=650$. The dash-dotted lines correspond to contours with at least one side outside IR with the dimensions $\left(\ell_{1} / \eta, \ell_{2} / \eta\right)$ as follows: (6.6,704), (11.0,422.4), (16.5,281.6), (22.0,211.2), (26.4,176.0), and $(33.0,140.8)$, in the direction of the arrows shown. The collapsed solid lines correspond to loops with both sides $\left(\ell_{1} / \eta\right.$, $\left.\ell_{2} / \eta\right)$ contained within IR, and correspond to $(44.0,105.6)$, $(52.8,88.0)$, and $(66.0,70.4)$. The collapsed curves in IR verify the area rule to a good approximation, more broadly than Migdal's anticipation for the tails (which drop off rapidly towards the extreme ends here because of finite sample sizes). The tails are approximately exponential in IR, especially for $\Gamma_{A}>0$, but are fitted better by stretched exponential fits. The parameters of the stretched exponential vary only slightly from one loop to another in IR.

figure supports the expectation that only the area of the loop, not its shape, decides the PDF of circulation for loops in IR. (Appendix B provides additional data expanding on this conclusion.)

We quantify the applicability and limitations of the area rule in Fig. 3, which plots the root mean square (rms) of circulation as a function of the loop area. The circulation standard deviation at $R_{\lambda}=1300$ pertains to two sets of contours with the same area but differing aspect ratios of 1 and 4 , as shown by the inset in the top left. For the two aspect ratios, distinguished by two different symbols, the data agree with each other, showing no major dependence on the aspect ratio. Their actual ratio, displayed in the lower right-hand inset, is close to unity within a few percent in IR and shows the nature of approximation one endures in invoking the area rule. Also shown in the inset is the similar ratio for $R_{\lambda}=240$. This smaller Reynolds number does not have an extensive IR, but there is a range of loop areas for which one may reasonably invoke the area rule. It is evident from Fig. 3 that, with increasing Reynolds number, the validity of the area rule extends to a wider range of scales.

The power law denoted by a dashed line in Fig. 3 is the classical K41 result, extended from the expectation that the velocity increments scale as $r^{1 / 3}$. The fit is very good-but with a slope that is slightly larger than $2 / 3$, as we shall examine more closely in Sec. IVA. 


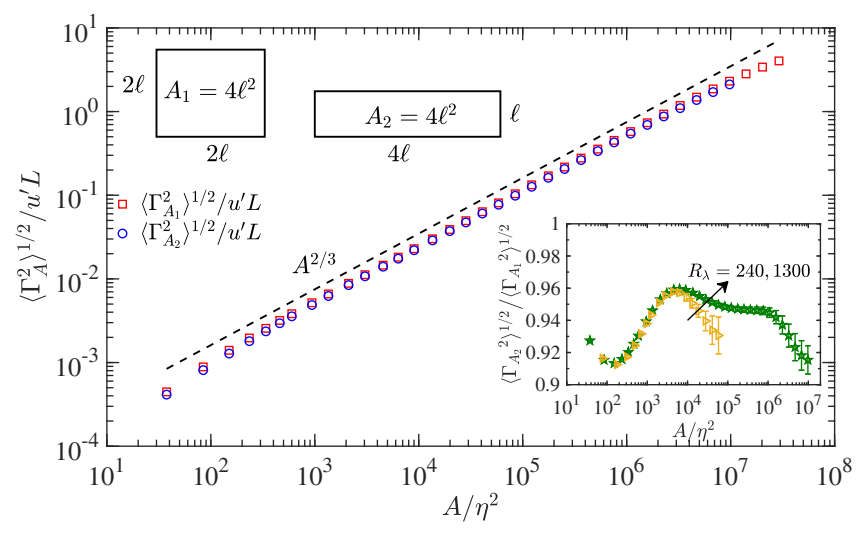

FIG. 3. Circulation standard deviation $\left\langle\Gamma_{A}^{2}\right\rangle^{1 / 2}$ for two sets of contours with the same loop area and different aspect ratios (see insets to the upper left) as a function of the area, at $R_{\lambda}=1300$, computed on a $8192^{3}$ grid. The standard deviations seem to be independent of the loop perimeter, especially for inertial loops (roughly, $10^{3} \leq A / \eta^{2} \leq 10^{6}$ ). Inset on the bottom right plots the ratio of the standard deviation of the two contours as a function of loop area. The ratio is quite close to unity, though smaller by a few percent. It is also not exactly a constant within IR. We also show the same ratio for a lower Reynolds number, $R_{\lambda}=240$. It is clear from the two Reynolds numbers that, within the accuracy just noted, the area rule holds increasingly well at higher Reynolds numbers. The dashed line indicates the K41 law, $\left\langle\Gamma_{A}^{2}\right\rangle^{1 / 2} \sim A^{2 / 3}$; it holds adequately for inertial loops though the actual least-squares fit gives a slope of 0.674 (which is about $1.2 \%$ higher).

In order to examine whether the circulation statistics around a loop depend on the scalar area enclosed by the loop or the tensor loop area [10], we consider a figure-eight loop as shown in the inset of Fig. 4, with two different squares with edge lengths $L_{1}$ and $L_{2}$ touching at a common vertex, with $L_{1}-L_{2}$ held as a fixed constant. If K41 is valid, it readily follows that the standard deviation of circulation based on the scalar area law will scale as $\left\langle\Gamma_{A}^{2}\right\rangle^{1 / 2} \sim$ $\left(L_{1}^{2}+L_{2}^{2}\right)^{2 / 3}=A^{2 / 3}$. On the other hand, if one traverses along the loop in the direction of the arrows marked on the loop in Fig. 4, the areas circumscribed by the two squares will have different signs. If the resulting vector area is the proper quantity to use, the standard deviation $\left\langle\Gamma_{A}^{2}\right\rangle^{1 / 2}$ for the figure-eight loop should scale as

$$
\left\langle\Gamma_{A}^{2}\right\rangle^{1 / 2} \sim\left(L_{1}^{2}-L_{2}^{2}\right)^{2 / 3}=\left[\Delta\left(L_{1}+L_{2}\right)\right]^{2 / 3} \sim A^{1 / 3},
$$

since $\Delta=L_{1}-L_{2}$ is a fixed constant. The main part of Fig. 4 shows convincingly that the mean-square circulation varies closely as $A^{2 / 3}$, where $A$ is the scalar sum of the areas of the two loops, for most of the range; it is certainly far from $1 / 3$. For small $A$, the variance is clearly linear in $A$, as expected from Taylor's expansion, whereas it saturates for large $A$ because of many cancellations. Although all the symbols collapse on each other quite well, a closer

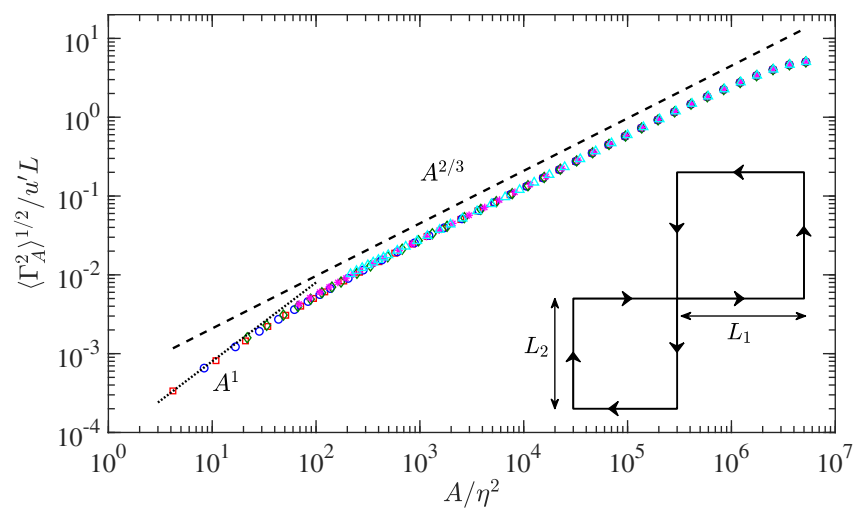

FIG. 4. Circulation standard deviation as a function of the area $A$ of the figure-eight loop (shown in the inset), calculated in a $4096^{3}$ grid at $R_{\lambda}=650$. The edge lengths of the figure-eight loop are chosen such that $L_{1}-L_{2}=\Delta$, where $\Delta$ is a fixed constant. Symbols (square), (circle), (diamond), (asterisk), and (triangle) correspond to $\Delta / \eta=2,4,8$, and 16 , respectively. The standard deviations collapse for different figure-eight loops with the same area and follow the scalar area rule in IR, as evidenced by the closeness to the $A^{2 / 3}$ scaling. The linear area dependence for small $A$ is shown, and the tendency to saturation for large areas is also apparent. Both these tendencies also occur in Fig. 3.

inspection reveals that the quality of this invariance is comparable to that in Fig. 3.

In summary, then, to a good approximation we find that the PDF of circulation depends essentially on the loop area, as long as both sides of the loops are contained within IR. It is worth emphasizing that we do not present this statement as exact but as a very good working approximation (more details will be presented elsewhere). We further find that intersecting loops, i.e., loops with common edges or vertices, such as the figure-eight loop, do not need any special attention, as the statistically relevant area is the scalar area enclosed by the loop. Thus, in the following, we use planar square loops for simplicity. It is then sometimes convenient to use the symbol $\Gamma_{A}$ to describe the circulation around a loop of area $A$; there is no ambiguity when we speak of $\Gamma_{r}$ because $r=A^{1 / 2}$.

\section{B. Kolmogorov's similarity argument for the third-order moment}

As stated already, straightforward application of K41 shows that $\left\langle\Gamma_{A}^{2}\right\rangle^{1 / 2} \sim A^{2 / 3}$; this is seen to be closely valid for inertial loops (see Figs. 2 and 3). In turbulence theory, a special place is held by the third-order moment of velocity increments, and so it would be instructive to examine the third-order moment of circulation as well. To assess Migdal's [9] proposal that the PDF of $\Gamma_{r}^{3} / r^{4}$ would be unique for all loops, we show the PDF of $\Gamma_{r}^{3} / r^{4}\langle\epsilon\rangle$ in Fig. 5 for various inertial loops. The PDFs show good collapse, with some modest deviations around $\Gamma_{r}^{3} / r^{4}\langle\epsilon\rangle= \pm 150$ and in the tails (see the two insets). Neither the positive nor the 


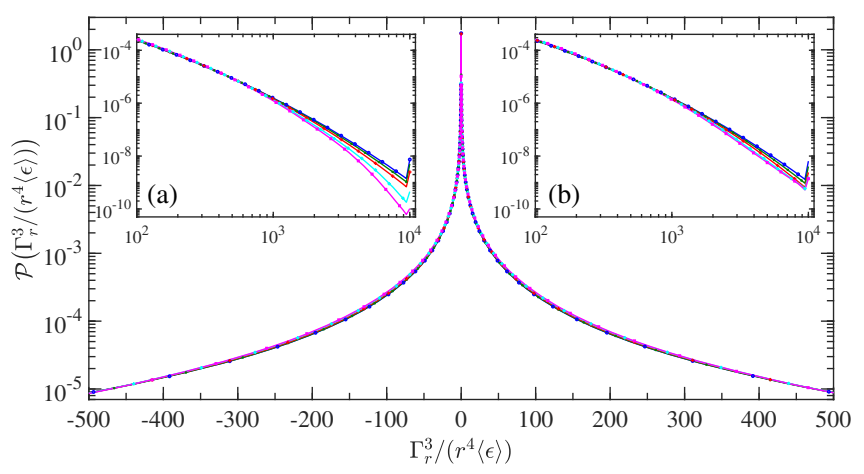

FIG. 5. PDF of $\Gamma_{r}^{3} / r^{4}\langle\epsilon\rangle$ at $R_{\lambda}=1300$ at different inertial range separations, $r / \eta=80$ (circle), $r / \eta=120$ (times), $r / \eta=171$ (plus), $r / \eta=246$ (asterisk), and $r / \eta=300$ (square). Insets (a) and (b) show portions of the PDF corresponding to negative and positive $\Gamma_{r}$, respectively, on log-log scales. The PDFs nearly collapse across the inertial range separations. If they are examined on an expanded scale, some modest differences are seen around $\Gamma_{r}^{3} / r^{4}\langle\epsilon\rangle= \pm 150$. Differences occur also towards the tails, as shown in the insets. The tails of each of the PDFs can be fitted by stretched exponentials with unequal stretching factors for the negative and positive tails. For the average curves, the negative part decays with a stretch factor of about 0.35 , about twice as large as that for the positive $(\approx 0.17)$.

negative tails of the PDF follow power laws. They can be fitted nominally by stretched exponentials with unequal stretching exponents, as stated in the caption of Fig. 5. The mean and the mean square of this distribution yield the third-order and sixth-order moments of circulation. These and other moments are computed separately in Sec. IVA.

Figure 5 suggests that K41 holds closely for the thirdorder circulation moment. We now explore the scaling properties of other orders.

\section{SCALING RESULTS FOR CIRCULATION MOMENTS}

\section{A. Exponents}

We first evaluate various even-order moments of $\Gamma_{r}$ and show in Fig. 6 moments $\left\langle\Gamma_{r}^{p}\right\rangle$ for $p=2,4,6$, and 8 for $R_{\lambda}=1300$ as functions of $r$. All of them display proper power laws in IR, $\left\langle\Gamma_{r}^{p}\right\rangle \sim r^{\lambda_{p}}$, as reinforced by the near constancy of local slopes presented in the inset of Fig. 6.

The odd moments of $\Gamma_{r}$ do not display equally clean power laws because they have negligible intensity via cancellation, leading to poor convergence. The scaling improves if one considers absolute values of circulation, $\left\langle\left|\Gamma_{r}\right|^{p}\right\rangle \sim r^{\lambda}|p|$ (while, obviously, those of even orders remain unchanged). Absolute values enable us to define scaling exponents for fractional $p$ as well [25] up to $p=-1$ (but not equal to -1 , for which the moments diverge). The practice of using absolute moments for odd orders is justified, at least a posteriori, as long as the exponents so obtained are monotonic when plotted together with even-order data.

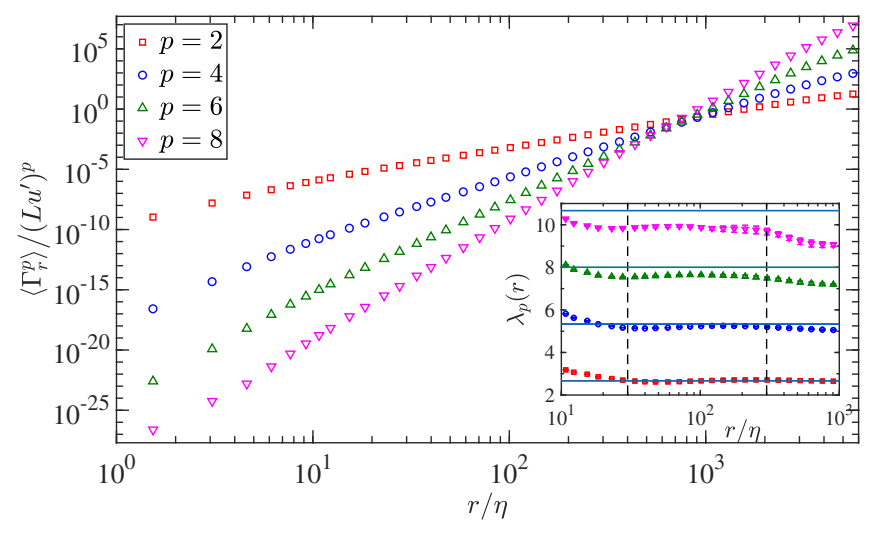

FIG. 6. Normalized circulation moments for various orders $p$ as a function of the linear dimension of the loop, for $R_{\lambda}=1300$. Inset shows corresponding local slopes $\lambda_{p}=d\left[\log \left\langle\Gamma_{r}^{p}\right\rangle\right] / d[\log r]$, with vertical lines demarcating the inertial range. Error bars (which are often subsumed by the symbol thickness) indicate $95 \%$ confidence intervals. Horizontal straight lines drawn at $4 p / 3$ correspond to K41 exponents.

In Fig. 7 we plot the scaling exponents $\lambda_{|p|}$ for circulation as a function of the power index $p$ for all orders at $R_{\lambda}=1300$. The exponents seem to organize themselves on two straight lines, one below $p=3$ and the other above 3 . The line for $p>3$ can be expressed by the fit

$$
\lambda_{|p|}=h p+(3-D)
$$

where $h=1.1 \pm 0.02$ and $D=2.2 ; h$ here is the Hölder exponent corresponding to $\left|\Gamma_{r}\right| \sim r^{h}$. This fit represents a monofractal [27] of dimension 2.2. The low-order data, $p<3$, can be fitted best by the straight line $\lambda_{|p|}=(1.367 \pm$ $0.0095) p$ with no intercept, suggesting that the moments reside on a space-filling set (dimension three); for simplicity we will call this combined behavior the 1.4 scaling (which is very close but not identical to K41, for which $\lambda_{|p|}=4 p / 3$ ). Inset (a) of Fig. 7 shows the expanded view of the region around $p=0$ to elucidate the space-filling nature at low orders.

In Sec. V, we develop the theme of two scaling regimes in greater detail. So far, we have considered results for only the two highest Reynolds numbers of our dataset. We shall also examine how the results depend on the Reynolds number, and assess the asymptotic state.

\section{B. Probability density functions}

We see in Fig. 5 that the PDFs of $\Gamma_{r}^{3} / r^{4}\langle\epsilon\rangle$ collapsed quite well for loops of different areas. The tails show some departure from the collapse, and a careful examination also reveals some departures for $\left|\Gamma_{r}^{3} / r^{4}\langle\epsilon\rangle\right|$ between 100 and 200, say. Nevertheless, at the level of third-order statistics, the collapse can be regarded as excellent, showing that K41 works quite well for this particular detail. 


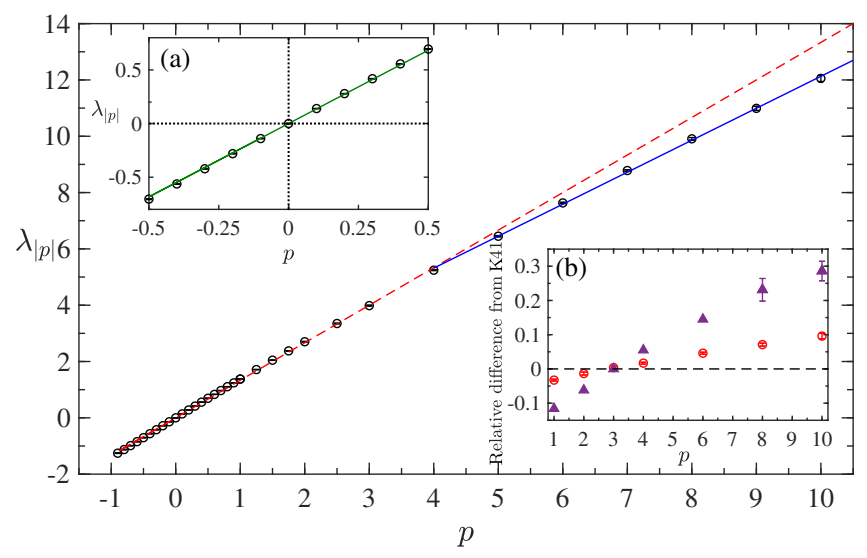

FIG. 7. Scaling exponents as a function of moment order $p$; $R_{\lambda}=1300$. The data can be fitted by two separate lines, one below order 3 and one above it. For comparison purposes, the dashed line is the $\mathrm{K} 41$ result given by $4 p / 3$. The low-order data can be fitted best by the expression $\lambda_{|p|}=(1.367 \pm 0.0095) p$ with no intercept as shown in inset (a) by the solid line. The highorder data can be fitted (solid line) by a monofractal model, $\lambda_{|p|}=1.1 p+(3-D)$, with $D=2.2$. Both fits are determined by least-squares method. The error bar shown for the tenth moment is typical and is subsumed by the symbol thickness. Inset (b) compares the relative departures of circulation exponents at $R_{\lambda}=1300$ and longitudinal velocity increment exponents (filled triangle) at $R_{\lambda}=10340$ [26], from their respective K41 estimates, for integral orders. The dashed line at zero is the K41 scaling. The relative deviations from $\mathrm{K} 41$ for higher-order circulation exponents are about a third of those of the longitudinal structure function exponents (but the sign of these differences in both cases is the same).

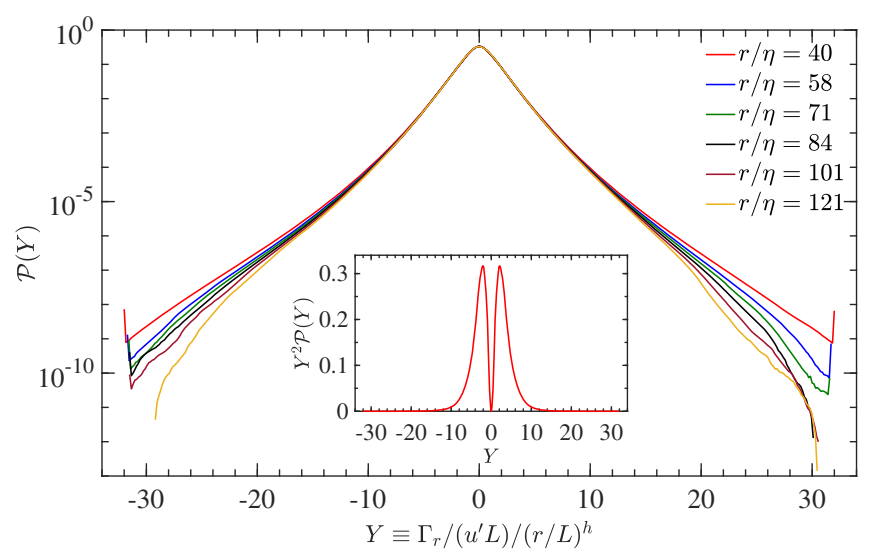

FIG. 8. Probability density function of circulation normalized by the $h \approx 1.4$ scaling for different inertial range separations, at $R_{\lambda}=1300$. The PDF cores collapse for $Y \in[-10,10]$ across the inertial range, while diverging for larger $|Y|$. Inset shows the integrand of $\left\langle Y^{2}\right\rangle$ for a typical inertial separation, showing that the dominant contribution to lower-order circulation moments comes from the region where the PDFs collapse. This plot confirms that the lower-order circulation moments follow the 1.4 scaling with the space-filling dimension of three.
That the 1.4 scaling works for all low-order moments can be seen by the collapse of the core region of the PDFs when normalized by $(r / L)^{1.4}$ for several values of $r$ in the inertial range. Figure 8 shows that the PDFs collapse onto each other, for amplitudes below about \pm 10 , which is decisively where low-order moments reside (as illustrated by the inset for the second moment). It should be emphasized that, even though K41-like similarity exists for low-order moments, the PDFs depart strongly from Gaussian.

But the PDFs of Fig. 5 differ in their tails, this being the seat of higher moments. If, as we claimed with respect to Fig. 7, the high-order moments reside on a fractal set of dimension $D=2.2$ (with corresponding Hölder exponent $h$ ), the tails of the PDF compensated by $r^{3-D}$ have to collapse when scaled on $r^{h}$. The corresponding result is shown in Fig. 9. The collapse of the tails beyond about 3 standard deviations is clear. Together, Figs. 8 and 9 reaffirm our claim that low-order moments follow a space-filling monofractal model, but the higher moments inhabit a fractal set of dimension 2.2. We discuss the potential significance of this inference in Sec. VA.

\section{Circulation flatness}

The circulation flatness is defined as

$$
F(r) \equiv \frac{\left\langle\Gamma_{r}^{4}\right\rangle}{\left\langle\Gamma_{r}^{2}\right\rangle^{2}}
$$

Figure 10 shows that $F(r)$ varies through the inertial range $(\eta \ll r \ll L)$, but its evolution with Reynolds number is the more interesting point; the figure also compares $F(r)$ at

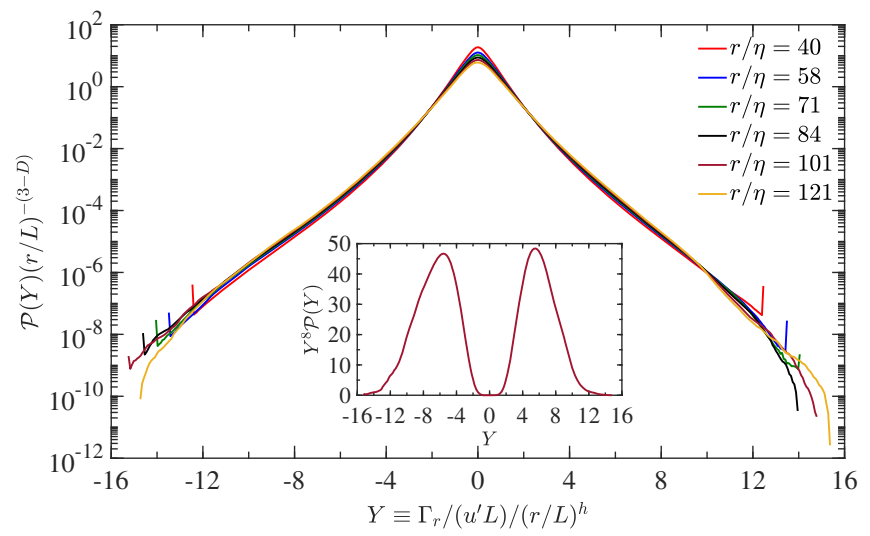

FIG. 9. Compensated probability density function of the normalized circulation $Y$, with scaling exponent $h=1.1$ and dimension $D=2.2$, for different inertial range separations, at $R_{\lambda}=1300$. The upticks at the tails of the PDFs are due to finite sampling. Inset shows the integrand of $\left\langle Y^{8}\right\rangle$ for a typical inertial separation, demonstrating that the dominant contribution to higher-order circulation moments comes from the collapsed region. This plot confirms that the higher-order circulation moments follow the scaling $\lambda_{p}=h p+(3-D)$, with the Hölder exponent $h=1.1$ and fractal dimension $D=2.2$. 


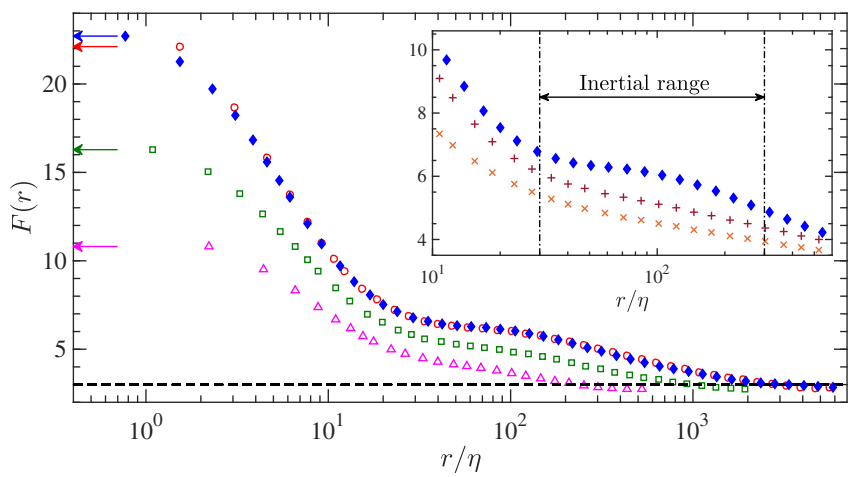

FIG. 10. Circulation flatness $F(r)$ versus spatial separation $r$ from DNS at $R_{\lambda}=240,512^{3}$ (triangle), $R_{\lambda}=650,4096^{3}$ (square), and $R_{\lambda}=1300,8192^{3}$ (circle). The flatness from the DNS at $R_{\lambda}=1300$ computed on a $16384^{3}$ box is also shown (filled diamond) and agrees well with $F(r)$ from the $8192^{3}$ box, for $r / \eta>10$. Arrows on the ordinate show the limit $F(r \rightarrow 0)=\left\langle\omega^{4}\right\rangle /\left\langle\omega^{2}\right\rangle^{2}$, which is the flatness of the vorticity component normal to the circulation plane. At the largest scales, $F(r)$ is close to the Gaussian flatness of three (see dashed line). Inset compares the circulation flatness with those of the longitudinal (times) and transverse (plus) velocity increments at $R_{\lambda}=$ 1300 in the inertial range within the dashed vertical lines. Unlike circulation with a tendency towards constancy in the inertial range, the velocity increments show no tendency towards constancy in IR.

three different Reynolds numbers. At $R_{\lambda}=240, F(r)$ increases smoothly from the Gaussian value of 3 , for $r \sim O(L)$, to the dissipation range limit of $\left\langle\omega^{4}\right\rangle /\left\langle\omega^{2}\right\rangle^{2}$, where $\omega$ is the vorticity component normal to the plane of circulation. With increasing $R_{\lambda}$, however, $F(r)$ tends towards proper scaling in IR, indicating that it may approach a constant that is independent of scale. The inset of Fig. 10 compares the circulation flatness to those of the longitudinal and transverse velocity increments at $R_{\lambda}=1300$. Here, the transverse increment $\Delta_{r} v \equiv$ $v(x+r)-v(x)$, where the separation distance $r$ is transverse to the velocity component $v$. Even at $R_{\lambda}=1300$, the flatness factors of both $\Delta_{r} u$ and $\Delta_{r} v$ smoothly grow with decreasing scale, showing that the velocity increments are highly intermittent, whereas $F(r)$ displays the tendency towards constancy, suggesting that $\Gamma_{r}$ at high Reynolds numbers is only weakly intermittent. It should be stated that, at lower $R_{\lambda}$, the flatness factors of all the three quantities, $\Gamma_{r}, \Delta_{r} u$, and $\Delta_{r} v$, increase rapidly with decreasing scale in the inertial range, as already shown in Refs. [13,15].

Our point from Fig. 10 is that the flatness $F(r)$ has the potential to become a constant in the inertial range as the Reynolds number increases further. If so, this will be the intermittency-free limit within which we expect the logarithmic local slope $d[\log F(r)] / d[\log r] \rightarrow 0$. To quantify this approach to the intermittency free limit, we plot the logarithmic local slope of $F(r)$ as a function of spatial

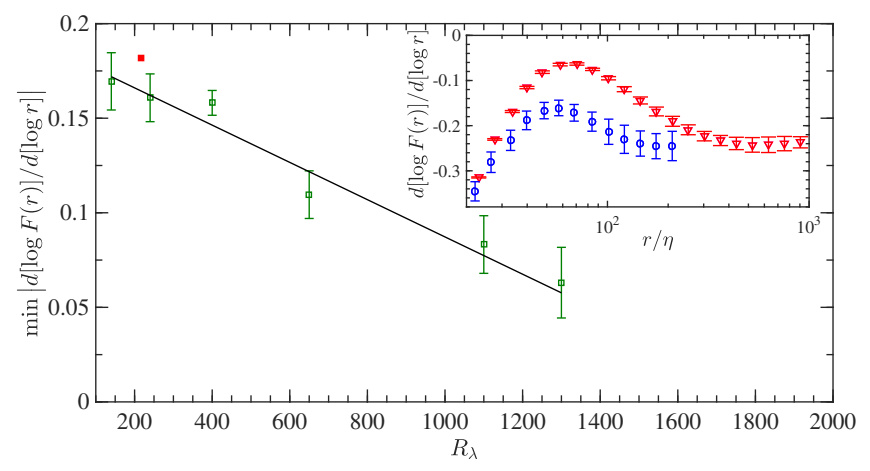

FIG. 11. The peak magnitude of logarithmic local slope of circulation flatness in the inertial range, plotted against $R_{\lambda}$. The solid line is a least-squares fit. If extrapolated, the minimum of the logarithmic derivative will reach zero, corresponding to constant flatness in Fig. 10 , at $R_{\lambda} \approx 1900$. The data of Ref. [13] at $R_{\lambda}=216$ (filled square) are shown for comparison. Inset shows the logarithmic local slopes of circulation flatness as a function of $r / \eta$, up to $r \sim O(L)$ for $R_{\lambda}=1300$ (upside down triangle) and $R_{\lambda}=240$ (circle). Error bars indicate $95 \%$ confidence intervals.

separation in the inset of Fig. 11 for two different Reynolds numbers. For $R_{\lambda}=1300$, the local slopes are closer to zero over a wider range of inertial scales than for $R_{\lambda}=240$. The peaks of the flatness local slopes decrease linearly with $R_{\lambda}$, as shown in Fig. 11, suggesting that it may become zero at $R_{\lambda} \approx 1900$. This result should be regarded merely as indicative and is discussed further in Sec. V B.

\section{DISCUSSION AND PERSPECTIVE}

Small-scale turbulence is known to be characterized by extreme events in time and space. Such intense events can be seen in local velocity increments and result in scaling exponents that vary nonlinearly with respect to the moment order. This is the phenomenon of intermittency that necessitates the superposition of infinitely many scaleinvariant configurations to describe it. Much effort has been expended in quantifying the nonlinear trend of the intermittency exponents of velocity moments, with varying degrees of success [8,28-32]. As noted in Ref. [33], intermittency corrections from the velocity moments serve as an upper estimate, since the velocity field is infrared divergent. Here, using the largest simulations of isotropic turbulence to date, we have shown that the structure of velocity circulation is much simpler at higher Reynolds numbers. In contrast to those of velocity increments, the circulation statistics at high Reynolds numbers has an approximately bifractal structure: for low moment orders, circulation is a space-filling quantity with the 1.4 scaling (close to but not equal to K41) whereas, for moments of orders above 3 , it has a self-similar dimension $D=2.2$ with a strictly sub-Kolmogorov scaling.

The inference that circulation, though arising from highly convoluted vortex structures in space and time, is 
space filling for low-order moments and resides on a fractal set of dimension 2.2 for high-order moments points to the existence of simplicity in its physics. This simplicity must manifest itself in the loop-space reformulation of the Navier-Stokes equations [10]. For instance, exact asymptotic formulas for vorticity correlations around fixed loops in the presence of large velocity circulation have recently been derived [34], and warrant further input from measurements. These observations by no means diminish the usefulness of the standard Navier-Stokes treatments (in physical space) which are better suited to studying other aspects of turbulence, such as the decay of turbulent kinetic energy [35].

Several questions arise from this work, but we discuss only a few of them here. (1) How definitive is the observation that low-order moments scale slightly differently from K41 (though very closely so)? What is the plausible significance of $D=2.2$ for high-order moments? (2) Since the data reveal that there is a Reynolds number dependence of the circulation properties, it is worth asking whether the asymptotic state has been reached even at the highest Reynolds number considered here, and, if not, making an educated guess on that state. This point is essentially an expansion of the tentative result deduced from Fig. 11. (3) Because the circulation is very closely related to vorticity, it is natural to ask why the simplicity that is apparent in circulation does not translate to enstrophy density, which is known to be a strongly multifractal quantity [36]. Finally, (4) what new research do these results suggest for the future? We discuss these questions in that same order.

\section{A. K41, low-order moments, and an inference from the high-order behavior}

We have experimented with plausible variations of the power-law fits from the one for which we have presented the results here. Considering that the differences between $\mathrm{K} 41$ and the 1.4 scaling are of the order of $3 \%$, it is impossible to escape the speculation that they may be some artifact of data processing (or even of the manner in which the data were generated). In fact, in an earlier version of this paper [41], we had stated that they were essentially K41. Our best assessment is that the low-order scaling for $p<3$ comes from a space-filling set whose Hölder exponent exceeds K41 by about 3\%. Since low-order circulation moments involve contributions from high probability events of low magnitude vorticities and low probability events of high magnitude but antiparallel vorticities, it is not surprising that this deviation from K41 exists. This slight negative anomaly $\left(4 p / 3-\lambda_{|p|}<0\right.$ for $\left.p<3\right)$ does not seem to diminish with increasing Reynolds number and appears to be a robust feature of circulation statistics.

Regarding the result that $D=2.2$ for higher moments, one may infer that they are due to moderately wrinkled vortex sheets rather than more complex singularities.

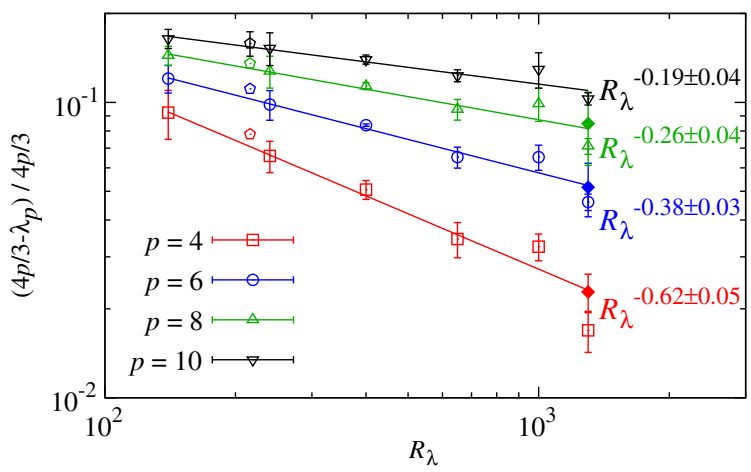

FIG. 12. The relative difference of the circulation scaling exponent $\lambda_{p}$ from the K41 exponent $4 p / 3$ as a function of the Taylor-scale Reynolds numbers $R_{\lambda}$, for orders $p=4,6,8$, and 10. Data from Ref. [13] (pentagon) for orders $p=4,6,8$, and 10, at $R_{\lambda}=216$, and the highly resolved DNS for orders $p=4,6$ and 8 , at $R_{\lambda}=1300,16384^{3}$ (filled diamond), are provided for comparison. Exponential least-squares fits through the data are shown by the solid lines. Also shown are error bars.

\section{B. Asymptotic state}

Figure 12 shows the behavior of $\delta_{\lambda_{p}}$, the relative difference between the measured scaling exponents and the corresponding K41 values, for moment orders 4, 6, 8, and 10, as functions of the Reynolds number. (Incidentally, we could have plotted the difference between the measured exponents and the 1.4 scaling, but the conclusions remain much the same.) For each order, this difference seems to approach K41 roughly as a power law. The rates of approach vary inversely with the order of the moment. We cannot speculate about the behaviors at infinitely large Reynolds numbers, but may expect that the behavior of Fig. 12 will persist up to some higher Reynolds number. That the approach to K41 is slower for the highorder moments suggests that, in principle, the scaling will remain a bifractal for all finite Reynolds numbers, with the "phase transition" point moving to a higher $p$ with increasing Reynolds numbers. In practice, however, moments may follow the 1.4 line for sufficiently high $p$ that the bifractal behavior may yield essentially a spacefilling monofractal.

\section{Circulation, enstrophy, and velocity increments}

Since circulation is very closely related to vorticity, it is natural to ask why the simplicity that is apparent in circulation does not translate to locally averaged enstrophy density, which is known to be a strongly multifractal quantity $[17,36]$. The corresponding question is relevant also with respect to velocity increments. We address this issue here briefly.

The area integral formula for circulation from Eq. (1) reads as

$$
\Gamma_{r}=\int \omega d A,
$$


where $\omega$ is the vorticity component perpendicular to the loop with area $A=r^{2}$ along an arbitrary direction. Raising both sides of the above equation to power $2 p$ and invoking Schwartz's inequality, we get

$$
\begin{gathered}
\Gamma_{r}^{2 p}=\left[\int \omega d A\right]^{2 p} \\
\leq\left[\int 1^{2} d A\right]^{p}\left[\int \omega^{2} d A\right]^{p} \\
=r^{2 p}\left[\int \omega^{2} d A\right]^{p} .
\end{gathered}
$$

Taking averages on both sides of the above inequality, we get

$$
\left\langle\Gamma_{r}^{2 p}\right\rangle \leq r^{2 p}\left\langle\left[\int \omega^{2} d A\right]^{p}\right\rangle .
$$

Now, define enstrophy, $\Omega \equiv \omega_{i} \omega_{i}$, and its local 2D average, $\Omega_{r} \equiv r^{-2} \int \Omega d A$, and use statistical isotropy to get for any power, $p \geq 1$,

$$
\left\langle\left(\int \omega^{2} d A\right)^{p}\right\rangle \leq \frac{1}{3}\left\langle\left(\int \Omega d A\right)^{p}\right\rangle=\frac{1}{3} r^{2 p}\left\langle\Omega_{r}^{p}\right\rangle,
$$

with equality valid for $p=1$. Substituting Eq. (11) in Eq. (10) we are led to

$$
\left\langle\Gamma_{r}^{2 p}\right\rangle \leq \frac{1}{3} r^{4 p}\left\langle\Omega_{r}^{p}\right\rangle
$$

Although the upper bound in the above inequality can, in principle, diverge to infinity in the limit $\nu \rightarrow 0$, inequality (12) is useful from an empirical viewpoint. This inequality shows that the moments of locally averaged enstrophy are likely to be substantially larger than those of circulation, and it is this that indicates that the multifractal character of enstrophy density need not necessarily carry over to circulation. In particular, for $p=1$, using the homogeneity condition, $\nu\left\langle\Omega_{r}\right\rangle=\left\langle\epsilon_{r}\right\rangle=\langle\epsilon\rangle$, and taking $\langle\epsilon\rangle>0$, we obtain

$$
\psi(r) \equiv \frac{\left\langle\Gamma_{r}^{2}\right\rangle \nu}{r^{4}\langle\epsilon\rangle} \leq \frac{1}{3}
$$

with equality occurring in the limit $r \rightarrow 0$,

$$
\lim _{r \rightarrow 0} \psi(r)=\lim _{r \rightarrow 0} \frac{\left\langle\Gamma_{r}^{2}\right\rangle \nu}{r^{4}\langle\epsilon\rangle}=\frac{\left\langle\omega^{2}\right\rangle \nu}{\langle\epsilon\rangle}=\frac{1}{3} \frac{\langle\Omega\rangle \nu}{\langle\epsilon\rangle}=\frac{1}{3} .
$$

Figure 13 shows that the DNS data satisfy inequality (13) for all $r$ and are consistent with the dissipative limit.

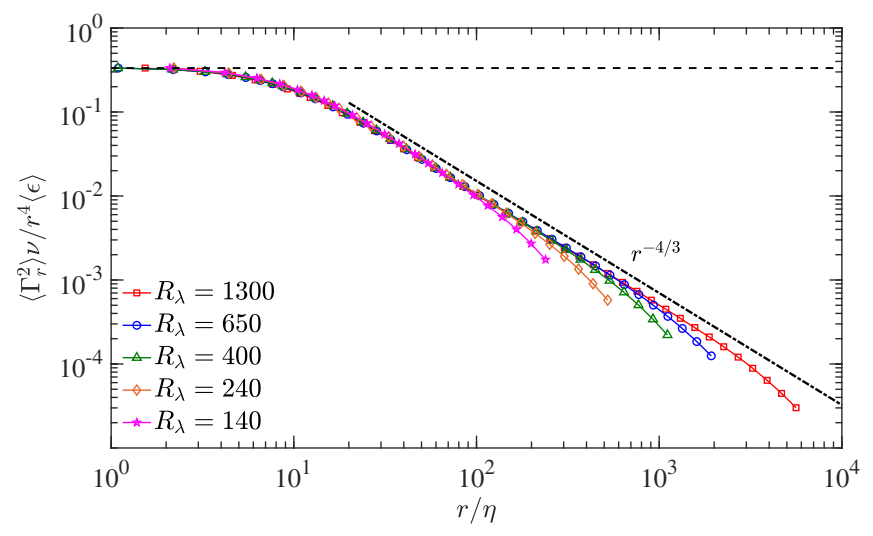

FIG. 13. The function $\psi(r)$ [left-hand side of inequality (13)] as a function of normalized spatial separation $r / \eta$ for different Reynolds numbers. The curves are seen to collapse, for $r \ll L$ ( $L$ is the integral scale), at the different Reynolds numbers shown, reasonably well. The limit $\psi(r \rightarrow 0)=1 / 3$ is marked by the horizontal dashed line. Dash-dotted line indicates the corresponding K41 slope.

Furthermore, $\psi(r)$ at different Reynolds numbers shows good collapse for $r \ll L$, where $L$ is the integral scale.

In order to relate the circulation moments to those of velocity increments, we invoke the gauge invariance property of circulation using the fact that $\oint_{C} d \mathbf{x}=\mathbf{0}$ around a closed contour $C$, with center $\mathbf{x}_{0}$ (say), to write

$$
\Gamma(C)=\oint_{C} d \mathbf{x} \cdot\left(\mathbf{u}(\mathbf{x})-\mathbf{u}\left(\mathbf{x}_{0}\right)\right) .
$$

Considering the magnitude of both sides of Eq. (15) and using the triangle inequality, we have

$$
|\Gamma(C)| \leq \oint_{C}\left|\mathbf{u}(\mathbf{x}(s))-\mathbf{u}\left(\mathbf{x}_{0}\right)\right| d s,
$$

where $s$ is the arc length along contour $C$ and $d s$ denotes an elemental length. Bounding the right-hand side using Hölder's inequality and then raising both sides of the inequality to power $p \geq 1$, we get

$$
|\Gamma(C)|^{p} \leq(L(C))^{p-1} \oint_{C}\left|\mathbf{u}(\mathbf{x}(s))-\mathbf{u}\left(\mathbf{x}_{0}\right)\right|^{p} d s,
$$

where $L(C)$ is the total length of the contour $C$. Now define the velocity structure function averaged in arc length $s$ along the loop $C$ as

$$
S_{p}(r(s))_{C}:=\frac{1}{L(C)} \oint_{C}\left|\mathbf{u}(\mathbf{x}(s))-\mathbf{u}\left(\mathbf{x}_{0}\right)\right|^{p} d s,
$$

where $\mathbf{r}(s)=\mathbf{x}(s)-\mathbf{x}_{0}, S_{p}(r)$ is the structure function of velocity increments, and the subscript $C$ denotes an average in arc length $s$ along the curve $C$. Since the loop $C$ is fixed in space with center $\mathbf{x}_{0}$, we must have 
$S_{p}(r)=V^{-1} \int S_{p}(r(s))_{C\left(\mathbf{x}_{0}\right)} d \mathbf{x}_{0}$ due to homogeneity, where $V=\int d \mathbf{x}_{0}$ is the volume. Physically, this corresponds to translating the loop $C$ through the entire volume $V$ and averaging. Finally, substituting Eq. (18) in inequality (17) and volume averaging, we end up with

$$
\left\langle|\Gamma(C)|^{p}\right\rangle \leq(L(C))^{p} S_{p}(r) .
$$

for $p \geq 1$. Now if $r$ is the characteristic length of the loop $C$, then $(L(C))^{p} \sim r^{p}$. Furthermore, assume $\left\langle|\Gamma(C)|^{p}\right\rangle=$ $G_{|p|}(r / L)^{\lambda_{|p|}}$ and $S_{p}(r)=B_{|p|}(r / L)^{\zeta|p|}$, for $\eta \ll r \ll L$, with exponents $\lambda_{|p|}$ and $\zeta_{|p|}$, respectively, where $(\cdot)_{|p|}$ denotes that $(\cdot)$ corresponds to absolute velocity increments and circulation. In general, the prefactors can depend on the Reynolds number as $G_{|p|} \sim R_{\lambda}^{\alpha_{|p|}}$ and $B_{|p|} \sim R_{\lambda}^{\beta_{|p|}}$ (for instance), where $\alpha_{|p|}$ and $\beta_{|p|}$ are order-dependent exponents. Then, inequality (19) can be recast in the limit $R_{\lambda} \rightarrow \infty$ (see Appendix C) as

$$
p+\zeta_{|p|} \leq \lambda_{|p|}+\frac{2}{3}\left(\beta_{|p|}-\alpha_{|p|}\right)
$$

In our DNS, we find that $\beta_{|p|}=0$ (nominally to four decimal places), whereas $\alpha_{|p|}<0$, i.e., circulation prefactors mildly decrease with Reynolds number, possibly due to cancellations in the integrands of Eq. (1). For $p>3$, since $4 p / 3>\lambda_{|p|}$ (from Fig. 7), the relative differences of $\lambda_{|p|}$ and $\zeta_{|p|}$ from their respective K41 values are related as

$$
\frac{p / 3-\zeta_{|p|}}{p / 3}>\frac{4 p / 3-\lambda_{|p|}}{4 p / 3}, \quad p>3,
$$

where we have followed the data in taking $\alpha_{|p|} / p \approx 0$ for $p>3$ and $\beta_{|p|} / p \approx 0$ for $p \geq 1$. Our data confirm this expectation convincingly for $p>3$ [see inset (b) of Fig. 7]. Thus, circulation exponents depart considerably less from their K41 values than do the exponents of velocity increments. These arguments show that the simplicity in the structure of circulation does not contradict the known multifractal properties of velocity increments or enstrophy density. We thus believe that we have covered new ground here.

\section{Perspective}

Some overall comments are now in order on where this work positions itself in the broad framework of turbulence research. The search for a unified scaling theory in turbulence has largely evolved around shell-averaged velocity increments [37,38] and ball-averaged energy dissipation and enstrophy [28,36], all of which display intermittency and nontrivial anomalous scaling. In contrast, by considering the Hopf generating functional which is known to satisfy a equation similar to the Schrödinger equation in quantum field theory, Migdal obtained a reduction of the three-parameter spatial dependence to a one-parameter dependence, namely the loop contour, in describing the generating functional for circulation. By traversing rectilinear loops in the inertial range at high Reynolds numbers, we have shown that circulation statistics depend, to a good approximation, on the loop area alone. At high Reynolds numbers, we have discovered that circulation correlations display weak anomaly and a much simpler scaling structure than other inertialrange quantities. The evidence presented here points to a reduction in complexity when one considers the average vorticity over loops, which seems to circumvent the spatial complexity involving velocity differences and gradients. This conclusion suggests a simplification of principle for the intermittency problem in 3D turbulence, when viewed through the lens of loop spaces. In doing so, we have helped foster the analogy first initiated by Migdal between Wilson loops in QCD and velocity circulation in turbulence [39]. It is not unreasonable to hope that this work could invigorate similar loop formulations in tackling turbulence.

In order to place the still nascent statistical theory of circulation on a firmer footing, a stringent examination is desirable of the circulation statistics for nonrectilinear loops, nonplanar loops, and even fractal loops. This work will be reported separately. Calculating quantities besides circulation, such as vorticity correlations around fixed loops, can shed light on the usefulness of loopbased approaches [34]. Finally, the results presented here can spur similar investigations of other problems which display anomalous scaling behavior, such as the magnetic field correlations in the kinematic dynamo problem [40], where the analog of circulation is the magnetic flux; one may anticipate similar simplifications in scaling theory.

\section{ACKNOWLEDGMENTS}

This work is partially supported by the National Science Foundation (NSF), via Grants No. ACI-1640771 and No. ACI-1036170 at the Georgia Institute of Technology. The computations were performed using supercomputing resources provided through the XSEDE consortium (which is funded by NSF) at the Texas Advanced Computing Center at the University of Texas (Austin), and the Blue Waters Project at the National Center for Supercomputing Applications at the University of Illinois (Urbana-Champaign). We thank Dr. Xiaomeng Zhai for his help in evaluating circulation via spline fits. This paper has benefited from discussions with Dr. A. A. Migdal, Dr. V. Yakhot, Dr. T. D. Drivas, Dr. A. M. Polyakov, Dr. E. D. Siggia, Dr. T. Spencer, and Dr. G. L. Eyink who provided valuable discussions and comments. We also thank the anonymous referees for useful comments. 


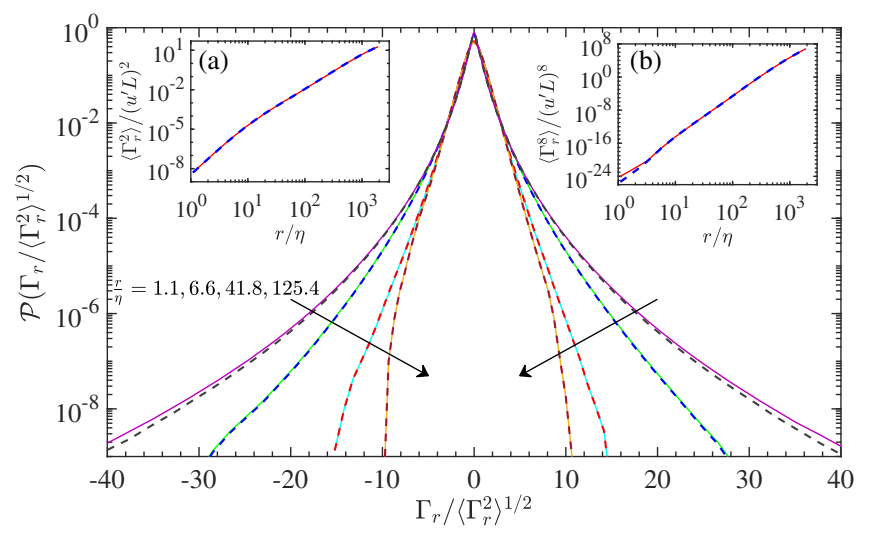

FIG. 14. Probability density function of circulation, normalized by its standard deviation, on a $4096^{3}$ grid at $R_{\lambda}=650$, calculated using [Eq. (A1)] line integral of velocity (dashed lines) and [Eq. (A2)] surface integral of vorticity (solid lines), around square loops of size $r$. Different sets of curves correspond to, $r / \eta=1.1$, 6.6, 41.8, and 125.4, increasing in the direction shown. Insets (a) and (b) compare the circulation moments from the two quadratures for orders 2 and 8 , respectively.

\section{APPENDIX A: STOKES THEOREM}

The circulation around a loop of linear dimension $r$ is defined as

$$
\Gamma_{r}:=\oint_{C(r)} \mathbf{u}(\mathbf{l}) \cdot d \mathbf{l}
$$

where $C(r)$ denotes the boundary of a loop of size $r$, with area $A(r), \mathbf{u}$ is the velocity, and $d \mathbf{l}$ is an elemental length along $C(r)$. Using the Stokes theorem, we can write

$$
\Gamma_{r}=\oiint_{A(r)} \omega \cdot \hat{\mathbf{n}} d A
$$

where $\omega \equiv \nabla \times \mathbf{u}$ is the vorticity and $\hat{\mathbf{n}} d A$ is an elemental area of $A(r)$. Figure 14 compares the statistics of $\Gamma_{r}$ calculated using the loop integration of Eq. (A1) and the area integral formula Eq. (A2). Excellent confirmation of the area integral results is obtained for $r / \eta>5$.

\section{APPENDIX B: AREA RULE}

With respect to the accuracy to which the area rule holds, we plot in Fig. 15 the peak value of the circulation probability density function for a fixed area but different aspect ratios, as a function of one side of the loop. (Since the area is fixed, a plot of the data against one side of the loop has the same information as that against the perimeter.) For contours with both dimensions in the inertial range, the PDF peaks decrease towards an approximately constant value in IR, shown by filled symbols, consistent with the area law.

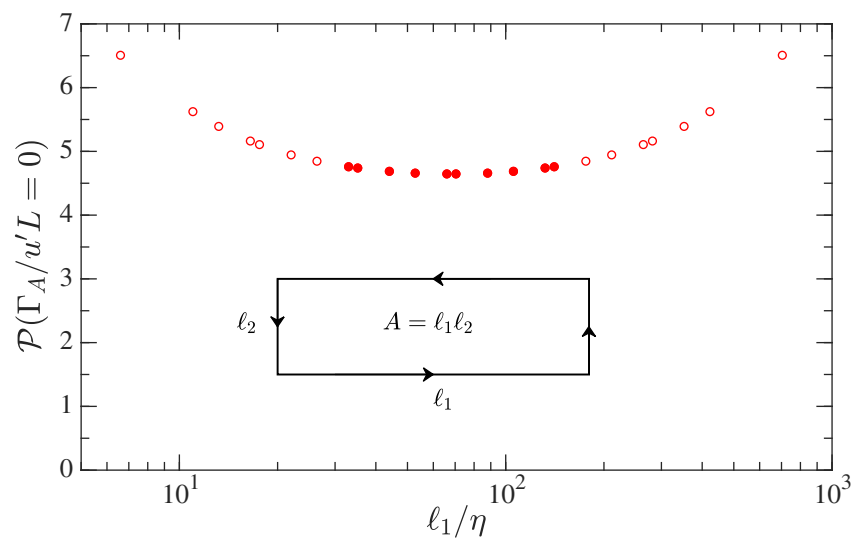

FIG. 15. Peak values of PDF of normalized circulation, $\Gamma_{A} / u^{\prime} L$, at $4096^{3}, R_{\lambda}=650$, around closed rectangular contours with fixed area, but different perimeters, $A=\ell_{1} \ell_{2}=4646.4 \eta^{2}$, as a function of side, $\ell_{1}$. The plot is symmetric around $\ell_{1}=\sqrt{A}$ due to statistical isotropy of the underlying velocity field (which holds by construction). Solid symbols correspond to contour dimensions $\left(\ell_{1}, \ell_{2}\right)$ with both legs in IR, while open symbols correspond to contours with at least one leg outside IR. The PDF peaks tend to an approximately constant value for inertial loops.

\section{APPENDIX C: RELATION BETWEEN CIRCULATION AND VELOCITY INCREMENT EXPONENTS}

Starting with inequality (19), which is valid for orders $p \geq 1$, assuming a power-law behavior for $p$ th-order moments of circulation along contour $C$ with characteristic size $r,\left\langle|\Gamma(C)|^{p}\right\rangle \equiv\left\langle\left|\Gamma_{r}\right|^{p}\right\rangle=G_{|p|}(r / L)^{\lambda_{|p|}}$ and velocity increments $S_{p}(r):=\left\langle|\mathbf{u}(\mathbf{x}+\mathbf{r})-\mathbf{u}(\mathbf{x})|^{p}\right\rangle=B_{|p|}(r / L)^{\zeta|p|}$ for $r$ in the IR, with exponents $\lambda_{|p|}, \zeta_{|p|}$ and prefactors $G_{|p|}, B_{|p|}$, respectively, we proceed as follows.

Let the prefactors of circulation and velocity increment moments vary with the Taylor-microscale Reynolds number as $G_{|p|} \sim R_{\lambda}^{\alpha_{|p|}}$ and $B_{|p|} \sim R_{\lambda}^{\beta_{|p|}}$, where $\alpha_{|p|}$ and $\beta_{|p|}$ are order-dependent exponents. Then, inequality (19) can be recast as

$$
(r / L)^{\lambda_{p}-p-\zeta|p|} \leq R_{\lambda}^{\beta_{|p|}-\alpha_{|p|}}
$$

Taking the logarithm on both sides of the inequality in the limit $R_{\lambda} \rightarrow \infty$ and noting that $r / L \rightarrow \eta / L$ in this limit, we get

$$
\left(\lambda_{p}-p-\zeta_{|p|}\right) \log (\eta / L) \leq\left(\beta_{|p|}-\alpha_{|p|}\right) \log R_{\lambda} .
$$

Using the K41 estimate $\eta / L \sim R_{\lambda}^{-3 / 2}$, dividing both sides of the above inequality by $\log R_{\lambda}$ in the limit $R_{\lambda} \rightarrow \infty$ and rearranging, we get

$$
p+\zeta_{|p|} \leq \lambda_{p}+\frac{2}{3}\left(\beta_{|p|}-\alpha_{|p|}\right) .
$$


TABLE II. Various terms in relation (C3) obtained using leastsquares fits for $8192^{3}$ DNS at $R_{\lambda}=1300$. Error bars are calculated as fluctuations in the inertial range local slopes around the least-squares fits, and correspond to $95 \%$ confidence intervals, obtained using a student's $t$ distribution.

\begin{tabular}{cccc}
\hline \hline$p$ & $p+\zeta_{|p|}$ & $\lambda_{|p|}$ & $2\left(\beta_{|p|}-\alpha_{|p|}\right) / 3$ \\
\hline 1 & $1.38 \pm 0.001$ & $1.38 \pm 0.004$ & $0.001 \pm 0.000$ \\
2 & $2.72 \pm 0.002$ & $2.70 \pm 0.01$ & $0.014 \pm 0.005$ \\
3 & $4.05 \pm 0.002$ & $3.98 \pm 0.02$ & $0.019 \pm 0.01$ \\
\hline \hline
\end{tabular}

By fitting power laws to the $p$ th-order moments $\left\langle\left|\Gamma_{r}\right|^{p}\right\rangle$ and $S_{p}(r)$ in the IR and using the large-scale matching $r / L \approx 1$ to obtain the prefactors, we find that $\beta_{|p|} \approx 0$, whereas $\alpha_{|p|}<0$, giving $\beta_{|p|}-\alpha_{|p|}>0$. Table II shows the various terms in inequality (C3) for orders $p=1,2$, and 3. Within the error bars of the calculations, the DNS results are consistent with inequality (C3), at least for orders $p \leq 10$.

[1] L. da Vinci, The Notebooks of Leonardo da Vinci, Pac PS, 2010.

[2] Y. Kaneda, T. Ishihara, M. Yokokawa, K. Itakura, and A. Uno, Energy Dissipation Rate and Energy Spectrum in High Resolution Direct Numerical Simulations of Turbulence in a Periodic Box, Phys. Fluids 15, L21 (2003).

[3] A. N. Kolmogorov, Local Structure of Turbulence in an Incompressible Fluid for Very Large Reynolds Numbers, Dokl. Akad. Nauk SSSR 30, 299 (1941).

[4] A. S. Monin and A. M. Yaglom, Statistical Fluid Mechanics (MIT Press, Cambridge, MA, 1975), Vol. 2.

[5] R. Benzi, G. Paladin, G. Parisi, and A. Vulpiani, On the Multifractal Nature of Fully Developed Turbulence and Chaotic Systems, J. Phys. A 17, 3521 (1984).

[6] U. Frisch, Turbulence (Cambridge University Press, Cambridge, England, 1995).

[7] G. L. Eyink, Besov Spaces and the Multifractal Hypothesis, J. Stat. Phys. 78, 353 (1995).

[8] K. R. Sreenivasan and R. A. Antonia, The Phenomenology of Small-Scale Turbulence, Annu. Rev. Fluid Mech. 29, 435 (1997).

[9] A. A. Migdal, Loop Equation and Area Law in Turbulence, Int. J. Mod. Phys. A 09, 1197 (1994).

[10] A. A. Migdal, Loop Equation and Area Law in Turbulence, in Quantum Field Theory and String Theory, edited by L. Baulieu, V. Dotsenko, V. Kazakov, and P. Windey, NATO ASI, Vol. 328 (Springer, Boston, 1995).

[11] M. Umeki, Probability Distribution of Velocity Circulation in Three-Dimensional Turbulence, J. Phys. Soc. Jpn. 62, 3788 (1993).

[12] K. R. Sreenivasan, A. Juneja, and A. K. Suri, Scaling Properties of Circulation in Moderate-Reynolds-Number Turbulent Wakes, Phys. Rev. Lett. 75, 433 (1995).

[13] N. Cao, S. Chen, and K. R. Sreenivasan, Properties of Velocity Circulation in Three-Dimensional Turbulence, Phys. Rev. Lett. 76, 616 (1996).
[14] R. Benzi, L. Biferale, M. V. Struglia, and R. Tripiccione, Self-Scaling Properties of Velocity Circulation in Shear Flows, Phys. Rev. E 55, 3739 (1997).

[15] Q. Zhou, C. Sun, and K. Q. Xia, Experimental Investigation of Homogeneity, Isotropy, and Circulation of the Velocity Field in Buoyancy-Driven Turbulence, J. Fluid Mech. 598, 361 (2008).

[16] P. K. Yeung, X. M. Zhai, and K. R. Sreenivasan, Extreme Events in Computational Turbulence, Proc. Natl. Acad. Sci. U.S.A. 112, 12633 (2015).

[17] P. K. Yeung, K. R. Sreenivasan, and S. B. Pope, Effects of Finite Spatial and Temporal Resolution in Direct Numerical Simulations of Incompressible Isotropic Turbulence, Phys. Rev. Fluids 3, 064603 (2018).

[18] V. Eswaran and S. B. Pope, An Examination of Forcing in Direct Numerical Simulations of Turbulence, Comput. Fluids 16, 257 (1988).

[19] D. A. Donzis and P. K. Yeung, Resolution Effects and Scaling in Numerical Simulations of Passive Scalar Mixing in Turbulence, Physica (Amsterdam) 239D, 1278 (2010).

[20] R.S. Rogallo, Numerical Experiments in Homogeneous Turbulence, NASA Technical Memo No. 81315, 1981.

[21] T. Ishihara, T. Gotoh, and Y. Kaneda, Study of HighReynolds Number Isotropic Turbulence by Direct Numerical Simulation, Annu. Rev. Fluid Mech. 41, 165 (2009).

[22] K. P. Iyer, Studies of Turbulence Structure and Turbulent Mixing Using Petascale Computing, Ph.D. thesis, Georgia Institute of Technology, 2014.

[23] K. P. Iyer, K. R. Sreenivasan, and P. K. Yeung, Reynolds Number Scaling of Velocity Increments in Isotropic Turbulence, Phys. Rev. E 95, 021101(R) (2017).

[24] A. N. Kolmogorov, Dissipation of Energy in Locally Isotropic Turbulence, Dokl. Akad. Nauk SSSR 434, 16 (1941).

[25] S. Y. Chen, B. Dhruva, S. Kurien, K. R. Sreenivasan, and M. A. Taylor, Anomalous Scaling of Low-Order Structure Functions, J. Fluid Mech. 533, 183 (2005).

[26] K. R. Sreenivasan and B. Dhruva, Is There Scaling in HighReynolds-Number Turbulence?, Prog. Theor. Phys. Suppl. 130, 103 (1998).

[27] B. B. Mandelbrot, Intermittent Turbulence in Self-Similar Cascades: Divergence of High Moments and Dimension of the Carrier, J. Fluid Mech. 62, 331 (1974).

[28] A. N. Kolmogorov, A Refinement of Previous Hypothesis Concerning the Local Structure of Turbulence in Viscous Incompressible Fluid at High Reynolds Number, J. Fluid Mech. 13, 82 (1962).

[29] C. Meneveau and K. R. Sreenivasan, A Simple Multifractal Cascade Model for Fully Developed Turbulence, Phys. Rev. Lett. 59, 1424 (1987).

[30] Z. S. She and E. Leveque, Universal Scaling Laws in Fully Developed Turbulence, Phys. Rev. Lett. 72, 336 (1994).

[31] V. Yakhot, Mean-Field Approximation and a Small Parameter in Turbulence Theory, Phys. Rev. E 63, 026307 (2001).

[32] D. Ruelle, Non-Equilibrium Statistical Mechanics of Turbulence, J. Stat. Phys. 157, 205 (2014).

[33] A. A. Migdal, Turbulence as Statistics of Vortex Cells, Selected Proceedings of the First Landau Institute Summer School, edited by V. P. Mineev (Gordon and Breach Publishers, London, 1995), pp. 177-204. 
[34] A. A. Migdal, Universal Area Law in Turbulence, arXiv: $1903.08613 v 3$.

[35] M. Sinhuber, E. Bodenschatz, and G. P. Bewley, Decay of Turbulence at High Reynolds Numbers, Phys. Rev. Lett. 114, 034501 (2015).

[36] C. Meneveau, K. R. Sreenivasan, P. Kailasnath, and M. S. Fan, Joint Multifractal Measures: Theory and Applications to Turbulence, Phys. Rev. A 41, 894 (1990).

[37] V. L'vov and I. Procaccia, The Universal Scaling Exponents of Anisotropy in Turbulence and Their Measurement, Phys. Fluids 8, 2565 (1996).
[38] S. Kurien and K. R. Sreenivasan, Measures of Anisotropy and the Universal Properties of Turbulence (Springer, Berlin, 2001), pp. 53-111.

[39] A. A. Migdal, Loop Equations and 1/N Expansion, Phys. Rep. 102, 199 (1983).

[40] M. Vergassola, Anomalous Scaling for Passively Advected Magnetic Fields, Phys. Rev. E 53, R3021 (1996).

[41] K. P. Iyer, K. R. Sreenivasan, and P. K. Yeung, Circulation in High Reynolds Number Isotropic Turbulence Is a Bifractal, arXiv:1902.07326. 